\title{
Detection of EGFR Mutation Distribution and Transcriptional Variants in IDH-Wildtype High-Grade Gliomas Using a Next- Generation Sequencing Oncopanel
}

\author{
Nayuta Higa \\ Kagoshima University: Kagoshima Daigaku \\ Toshiaki Akahane \\ Kagoshima University: Kagoshima Daigaku \\ Taiji Hamada \\ Kagoshima University: Kagoshima Daigaku \\ Hajime Yonezawa \\ Kagoshima University: Kagoshima Daigaku \\ Hiroyuki Uchida \\ Kagoshima University: Kagoshima Daigaku \\ Ryutaro Makino \\ Kagoshima University: Kagoshima Daigaku \\ Shoji Watanabe \\ Kagoshima University: Kagoshima Daigaku \\ Tomoko Takajo \\ Kagoshima University: Kagoshima Daigaku \\ Seiya Yokoyama \\ Kagoshima University: Kagoshima Daigaku \\ Mari Kirishima \\ Kagoshima University: Kagoshima Daigaku \\ Shingo Fujio \\ Kagoshima University: Kagoshima Daigaku \\ Ryosuke Hanaya \\ Kagoshima University: Kagoshima Daigaku \\ Akihide Tanimoto \\ Kagoshima University: Kagoshima Daigaku \\ Koji Yoshimoto ( $\sim$ kyoshimo@m.kufm.kagoshima-u.ac.jp ) \\ Kagoshima University: Kagoshima Daigaku https://orcid.org/0000-0001-7912-2024
}

\section{Research Article}

Keywords: glioblastoma, EGFR variant, EGFRv『, NGS, EGFR kinase domain

Posted Date: December 15th, 2021

DOI: https://doi.org/10.21203/rs.3.rs-861386/v2

License: (c) (1) This work is licensed under a Creative Commons Attribution 4.0 International License. Read Full License 


\section{Abstract}

\Purpose: To detect the epidermal growth factor receptor gene (EGFR) mutation profile and transcriptional variants in high-grade gliomas (HGGs), we sequenced EGFR and evaluated the EGFR splicing profile using a next-generation sequencing (NGS) oncopanel.

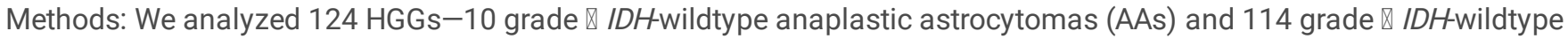
glioblastomas (GBMs).

Results: The EGFR mutations were observed in $6.0 \%$ of grade $\triangle \mathrm{GBMs}$ and in $33 \%$ of grade $₫ \mathrm{AAs}$. Four cases harbored missense mutations in the EGFR kinase domain (L747A, S768I, V774M, and T790M). A total of 25\% of the GBMs showed EGFR amplification. Moreover, $27 \%$ of the EGFR mutations occurred in the kinase domain. EGFRV GBMs. We identified two other EGFR variants in GBM cases with deletions of exons 6-7 ( $\triangle \mathrm{e} 6-7$ ) (one case) and exons 2-14 ( $\triangle \mathrm{e} 2-$ 14) (two cases). Interestingly, in one case, the initial EGFRVIII mutation transformed into an EGFR $\triangle$ e 2-14 mutation during recurrence. The frequency of EGFR alterations in our cohort was lower but the frequency of EGFR mutations in the kinase domain in our cohort was higher than that in The Cancer Genome Atlas and Memorial Sloan Kettering Cancer Center cohorts.

Conclusions: We suggested that the EGFR gene profiles of GBM differ among cohorts and identified rare EGFR variants with longitudinal and temporal transformations of EGFRvख.

\section{Introduction}

The epidermal growth factor receptor gene (EGFR) is the most commonly amplified and overexpressed proto-oncogene in glioblastoma multiforme (GBM) and causes the most malignant subtype. EGFR amplification is detectable by next-generation sequencing (NGS) in approximately $40-50 \%$ of GBM cases [1-3], and $14.4-26 \%$ of GBMs harbor EGFR mutations [2, 3]. Recently, the Consortium to Inform Molecular and Practical Approaches to CNS Tumor Taxonomy (CIMPACT-NOW) proposed a diagnostic entity of grade II-III isocitrate dehydrogenase (IDH)-wildtype astrocytoma that should behave similar to GBM. The molecular features defining this new tumor entity include telomerase reverse transcriptase promoter (TERTP) mutation, EGFR amplification, or a combination of whole chromosome 7 gain and whole chromosome 10 loss [4]. EGFR may serve as a diagnostic and prognostic biomarker. Indeed, EGFR has garnered interest as a drug target in GBM because of the high frequency of EGFR alterations in this disease. However, GBMs respond poorly to EGFR inhibitors [5, 6]. As EGFR mutations in GBM occur in the extracellular domain rather than the intracellular kinase domain, EGFR inhibitors that target the kinase domain are less effective [6].

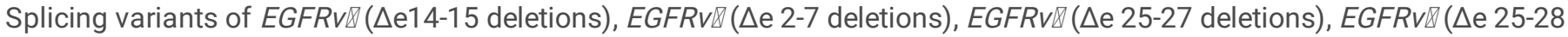
deletions), and $\operatorname{EGFR}(\triangle \mathrm{e} 12-13$ deletions) have been reported in $\operatorname{GBMs}[2,3,7,8]$. $E G F R V$, which is generated by the fusion of exons

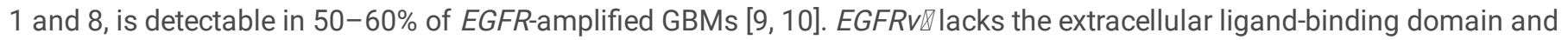
constitutively activates the receptor tyrosine kinase domain that increases GBM cell proliferation, migration, and invasion [11, 12]. $E G F R v \otimes$ may serve as a tumor-specific target for anti-EGFRv囚 immunotherapy, including antibody-based approaches and vaccines, such as rindopepimut, which produces a survival signal when combined with bevacizumab in EGFRv\&-positive recurrent GBMs [13]. However, immunotherapy failed in a phase III clinical trial in newly diagnosed patients [14]. This may be due to the frequent loss of EGFRV\expression with standard chemoradiotherapy.

To evaluate the EGFR mutational and splicing profile in IDH-wildtype high-grade gliomas (HGGs), we performed NGS analysis using a custom gene panel that we recently reported [15]. In this study, we collected a higher number of samples to clarify EGFR mutational distribution and developed a method to detect EGFR transcriptional variants.

\section{Materials And Methods}

\section{Diffuse glioma samples}

One hundred and twenty-four formalin-fixed paraffin-embedded (FFPE) tumor tissue samples were selected from the Central Nervous System Tumor Tissue Bank at Kagoshima University Hospital. The study was approved by the Institutional Review Board of Kagoshima University (approval number: 180104) and complied with the tenets of the Declaration of Helsinki. Informed consent was obtained from all patients. Resected tumors were fixed with phosphate-buffered $10 \%$ formalin within $24 \mathrm{~h}$ of sampling and routinely 
processed for paraffin embedding, followed by sectioning for hematoxylin and eosin staining. All tumors were originally classified according to the World Health Organization classification of 2016. The tumor series consisted of 10 grade III IDH-wildtype anaplastic astrocytomas (AAs) and 114 grade IV IDH-wildtype GBMs. All tissues were histologically evaluated by board-certified pathologists (M.K. and A.T.) to ensure an estimated tumor cell content of $30 \%$ or more. In all patients, when analyzing copy number variations, we sequenced leukocyte DNA for comparison against matched tumor DNA.

\section{DNA extraction and quantification}

For DNA preparation from FFPE samples, we used the Maxwell 16 FFPE Tissue LEV DNA Purification kit (Promega) according to the manufacturer's instructions. Thereafter, the concentration of DNA was measured using a Qubit 3.0 Fluorometer dsDNA BR Assay kit (Life Technologies), and DNA quality was monitored using the QIAseq DNA QuantiMIZE kit (QIAGEN). The extracted DNA was diluted to a concentration of $5-10 \mathrm{ng} / \mu \mathrm{L}$ as a template, and polymerase chain reaction (PCR) was performed using the QIAseq DNA QuantiMIZE kit.

\section{Next-generation sequencing}

NGS was performed using an amplicon-based glioma-tailored gene panel as described previously [15]. Amplicon sequences were aligned to the human reference genome GRCh37 (hg19) in the target region of the sequence. Data were analyzed using the QIAGEN Web Portal service (https://www.qiagen.com/).

\section{Detection of EGFR variants}

In all patients, to analyze rearrangement events as significant targets of intragenic copy number breakpoints, we sequenced leukocyte DNA for comparison against matched tumor DNA. The EGFR variants were detected by reduced read counts obtained for EGFR sequences in tumor DNA relative to those in matched leukocyte DNA. In addition, as a validation method, the EGFR variants were detected by determining the copy number $(\mathrm{CN})$ of each exon in EGFR and identifying exon sites with -2SD below in CN compared with the average $\mathrm{CN}$ of all exons.

\section{Complementary DNA analyses of clinical specimens}

The total RNA from FFPE samples of clinical specimens was extracted using the Maxwell 16 LEV RNA FFPE Purification kit (Promega) and converted into cDNA using the ReverTra Ace qPCR RT Kit \& Master Mix (TOYOBO Inc., Osaka, Japan). The cDNA fragments around the targeted site were amplified by PCR using the KOD One PCR Master Mix (TOYOBO Inc.) using the primers listed in Supplementary Table S1. The PCR products were analyzed by electrophoresis on $2 \%$ agarose gels. The PCR fragments were purified using the Exo-CIP Rapid PCR Cleanup kit (New England Biolabs Inc., Ipswich, MA) and analyzed by GENEWIZ Japan Corp.

\section{Immunohistochemistry}

Surgical specimens were fixed within 10 min of excision in $10 \%$ neutral buffered formaldehyde for $24 \mathrm{~h}$, embedded in paraffin, cut into 3- $\mu \mathrm{m}$-thick sections, and mounted on glass slides coated with poly-l-lysine. Subsequently, the sections were probed with 1:25

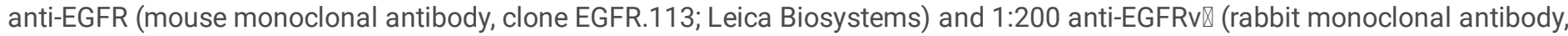
cat\#64952; Cell Signaling Technology). The sections were then stained with diaminobenzidine tetrahydrochloride and hematoxylin.

\section{TCGA and MSKCC data analyses}

We retrieved the molecular characteristics of the GBM cohort from previous publications. After excluding $H 3 F 3 A$, IDH1/2, and $B R A F$ V600E-mutant cases, we analyzed 343 cases from The Cancer Genome Atlas (TCGA) cohort and 456 cases from the Memorial Sloan Kettering Cancer Center (MSKCC) cohort [16]. All cases were conclusively diagnosed as IDH-wildtype GBM using the cBioPortal for Cancer Genomics (https://cbioportal.org).

\section{Definition of actionable alterations}

Actionable gene alterations were predicted to confer sensitivity to either an approved targeted agent or an experimental targeted agent currently under clinical trials. The JAX Clinical Knowledgebase (JAX-CKB) (https://ckb.jax.org/) was used to identify clinically actionable variants. 


\section{Data analysis}

We used OncoPrinter (cbioportal.org/oncoprinter) and MutationMapper (cbioportal.org/mutation_mapper), which are tools in the cBioPortal for Cancer Genomics, to visualize and analyze our data $[17,18]$. Statistical analyses were performed using JMP Pro v13 software (SAS Institute, Cary, NC). Groups were compared using the $\chi^{2}$ test. Differences were considered significant at $p<0.05$.

\section{Results}

\section{EGFR alterations in HGGs}

We analyzed $124 \mathrm{HGGs}-10 \mathrm{IDH}$-wildtype AAs and $114 \mathrm{IDH}$-wildtype GBMs. EGFR alterations were detected in 25\% of IDH-wildtype GBMs (Fig. 1a)and $60 \%$ of IDH-wildtype AAs (Fig. 1a). The EGFR mutation frequency in IDH-wildtype AAs was higher than that in IDHwildtype GBMs ( $50 \%$ and $6.1 \%$, respectively; $p<0.001$ ) (Table 1 ). Three cases had 2 EGFR point mutations and one case had 3 EGFR point mutations (Table 1). We also identified mutations in the EGFR extracellular and intracellular domains in 64.7\% (11/17 mutation sites) and 35.3\% (6/17 mutation sites) HGGs, respectively (Fig. 1b). Four cases harbored missense mutations in the EGFR kinase domain (encoding L747A, S768I, V774M, and T790M) (Table 1). In GBMs, 27\% (3/11 mutation sites) EGFR mutations were kinase domain mutations (Fig. 1b), and 18.2\% (2/11 mutation sites) EGFR mutations were druggable and registered in the JAX-CKB database (Fig. 1C). Furthermore, 20\% patients with IDH-wildtype AA and 1.8\% of patients with IDH-wildtype GBM harbored druggable mutations registered in the JAX-CKB database (Table 1). Additionally, 50\% patients with IDH-wildtype AA and $21.9 \%$ patients with IDH-wildtype GBM had EGFR amplifications.

\section{EGFR variants in HGGs}

The EGFRVQ $(\triangle 2-7)$ variant was detected by NGS in three (1 AA and $2 \mathrm{GBMs}$ ) of the 30 tumors with EGFR amplification (Table 2). All EGFRV\-positive cases were verified by immunohistochemistry and reverse transcription-PCR (RT-PCR) (Fig. 2a, d). EGFRv》was detected in two of the 25 EGFR-amplified GBMs (8.0\%). In addition, we identified two other EGFR variants in GBM cases, namely deletions of exons 6-7 ( $\triangle \mathrm{e}$ 6-7) and exons 2-14 ( $\triangle \mathrm{e}$ 2-14). EGFR $\triangle \mathrm{e}$ 6-7 was detected in 1 case, and $E G F R \triangle \mathrm{e} 2-14$ was detected in 2 cases (Table 2). Both EGFR $\triangle \mathrm{e}$ 6-7 and EGFR $\triangle \mathrm{e}$ 2-14 were verified by RT-PCR (Fig. 2b, c, d). All EGFR variants showed EGFR amplification (Table 2).

\section{Longitudinal and temporal transformation of EGFRv凹}

As an example, a 57-year-old woman with complaints of seizures underwent resection for a lesion in the left temporal lobe with high FLAIR signals observed by magnetic resonance imaging and high methionine accumulation observed by positron emission tomography. The pathological diagnosis was IDH-wildtype AA with an $E G F R V \otimes(\triangle \mathrm{e} 2-7)$ variant (Fig. 3a). Two years later, a tumor recurred in the same area, and the patient underwent another resection. The pathological diagnosis was IDH-wildtype GBM with

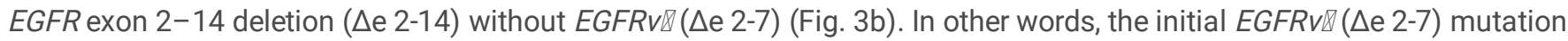
transformed into EGFR exon 2-14 deletion ( $\triangle \mathrm{e}$ 2-14), with extensive deletion of exons 8-14 at the time of recurrence.

\section{Frequencies of genetic alterations differed among the cohorts}

The frequency of EGFR alterations in our cohort (Fig. 1a) was lower than that in TCGA cohort ( $25 \%$ and $57 \%$, respectively; $p<0.001)$ and MSKCC cohort ( $25 \%$ and $44 \%$, respectively; $p<0.001)$. However, the frequency of EGFR mutations in the kinase domain in our cohort (Fig. 1a) was higher than that in TCGA cohort ( $27 \%$ and $5.9 \%$, respectively; $p=0.0401)$ and MSKCC cohort $(27 \%$ and $4.1 \%$, respectively; $p=0.0134$ ) (Supplementary Fig. 1 ).

\section{Discussion}

In this study, we collected higher number of samples than in our previous study [15]. We found that the frequency of EGFR mutations was low but the frequency of EGFR mutations in the kinase domain was high in wildtype-GBMs. Moreover, we developed a method to detect EGFR transcriptional variants; we found a novel EGFR transcriptional variant and identified a case with a rare EGFR variant with longitudinal and temporal transformations of EGFRv凹. 
EGFR amplification is detectable in approximately $40-50 \%$ of all GBMs [1-3]. The frequency of EGFR alterations in our cohort was lower than that in TCGA and MSKCC cohorts. However, a study from Japan reported that $25.5-33.1 \%$ of GBMs have EGFR amplifications [19,20], which is consistent with our findings. Recent reports indicate that $14.4-26 \%$ GBMs harbor EGFR mutations [2, 21]; however, the EGFR mutation frequency in GBMs in our cohort (6.0\%) was considerably lower than that in the previous study cohort. These results suggest that EGFR alterations may be less frequent in Japan than in other countries. Moreover, in our study, $E G F R^{A 289 D / T / V}$ was the most common missense mutation, and two cases harbored $E G F R^{T 790 M}$ missense mutations in the EGFR kinase domain. Previous studies have shown that approximately 4\% EGFR mutations in GBM have mutations in the kinase domain $[2,21]$; however, in our study, $27 \%$ EGFR mutations were in the kinase domain in GBMs, and this is higher than that reported previously.

Two cases (1 GBM and $1 \mathrm{AA}$ ) harbored $E G F R^{T 790 M}$ missense mutations in the kinase domain. $E G F R^{T 790 M}$, which is commonly observed in lung cancer [22], is a very rare mutation in glioma, with only one case of GBM reported previously [23]. Osimertinib is an oral, third-generation tyrosine kinase inhibitor (TKI) that irreversibly inhibits EGFR and was developed specifically to target the

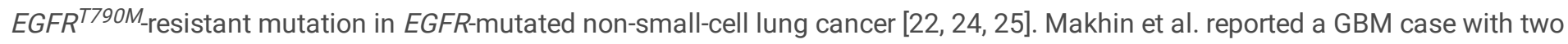
EGFR point mutations ( $\mathrm{C} 628 \mathrm{~F}$ and $\mathrm{A} 289 \mathrm{~V}$ ) that responded well to osimertinib. Thus, glioma cases with mutations in the EGFR kinase domain may benefit from EGFR TKIs [26]. In our study, a high EGFR amplification was identified in $5 / 10$ (50\%) patients with IDHwildtype $\mathrm{AA}$, consistent with the findings of a previous study [27]. In patients with IDH-wildtype AA, $33 \%$ EGFR mutations $(2 / 6$ mutation sites) had T790M and S768I mutations in the kinase domain. However, in IDH-wildtype AA, the distribution of mutations within the EGFR coding sequence has not been reported.

EGFRV\is the most common EGFR splice variant. EGFRV \activates multiple downstream signaling pathways and exhibits high

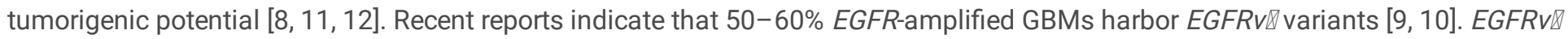
positivity was detected in 2 of the 25 EGFR-amplified glioblastomas (8.0\%) in our study, suggesting that EGFRV\positivity may have been less frequent in our study than in previous studies. These discrepancies may be due to ethnic differences in patient cohorts or differences in analysis methods; we used NGS and not immunohistochemistry or RT-PCR. A comparative study found that RT-PCR was more sensitive and specific than immunostaining using two different EGFRvख-specific antibodies [28]. Another study showed

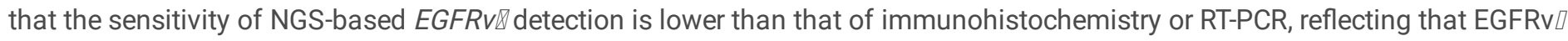
may be restricted to small subclones of glioma cells, which may not lead to a detectable reduction in exon 2-7 gene dosage [29].

In this study, we identified one uncharacterized EGFR variant with deletions of exons 6-7 ( $\triangle \mathrm{e} 6-7)$. Deletions of exons 2-14 ( $\triangle \mathrm{e}$ 2-14) constitute a very rare variant in glioma, with only one case of GBM having been reported previously [30]. In one case, the initial

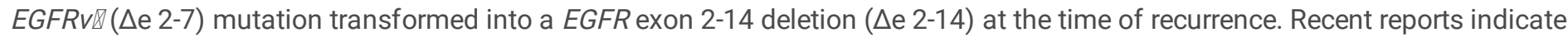

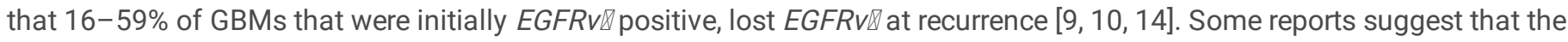

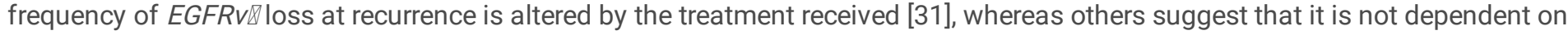
the treatment received [14]. In addition, it has been reported that the expression of EGFRV\is a result of epigenetic regulation [32], but the mechanism by which EGFRV囚 is lost at recurrence is unknown. Based on our findings, it is possible that the initial EGFRV mutation is transformed to other variants during recurrence. The functional significance of the novel EGFR variants needs to be analyzed in further studies.

In conclusion, we report the distribution of mutations within the EGFR coding sequence and two novel EGFR variants, one of which showed longitudinal and temporal transformation of EGFR $V$. In addition, we showed that the frequency of driver gene alterations in GBMs differs across cohorts. Thus, to implement personalized medicine, it is necessary to accurately assess the genetic profile of each cohort.

\section{Declarations}

\section{Funding}

The authors received no financial support related to this study.

\section{Conflicts of interest/Competing interests}

The authors declare that they have no conflict of interest. 
Availability of data and material

All data used and analysed in the current study are available from the corresponding author on reasonable request.

\section{Code availability}

Not applicable.

\section{Authors' contributions}

Conception and design: AT, KY; development of methodology: TA, TH, SY, TT; acquisition of data: NH, HY, HU, MK, RM, SW, SF; analysis and interpretation of data: $\mathrm{NH}, \mathrm{TA}, \mathrm{TH}, \mathrm{SY}$; manuscript writing, review, and/or revision: $\mathrm{NH}, \mathrm{RH}, \mathrm{AT}, \mathrm{KY}$; administrative, technical, or material support (e.g., reporting or organizing data and constructing databases): NH, TT, HY, HU, TA, TH, SY; study supervision: $\mathrm{RH}, \mathrm{AT}, \mathrm{KY}$.

\section{Ethics approval}

The study was approved by the Institutional Review Board of Kagoshima University (approval number: 180104) and complied with the tenets of the Declaration of Helsinki.

\section{Consent to participate}

Informed consent was obtained from all patients

\section{Consent for publication}

All authors agreed to the publication of the manuscript.

\section{Acknowledgments}

The authors thank Ippei Sakamoto and Sachio Nohara (Mitsubishi Space Software Co., Ltd.) for technical assistance in analyzing EGFR variants.

\section{References}

1. Weller M, Kaulich K, Hentschel B et al (2014) Assessment and prognostic significance of the epidermal growth factor receptor vIll mutation in glioblastoma patients treated with concurrent and adjuvant temozolomide radiochemotherapy: EGFRvIII mutation and prognosis of glioblastoma. Int J Cancer 134:2437-2447

2. Brennan CW, Verhaak RGW, McKenna A et al (2013) The somatic genomic landscape of glioblastoma. Cell 155:462-477

3. Francis JM, Zhang C-Z, Maire CL et al (2014) EGFR variant heterogeneity in glioblastoma resolved through single-nucleus sequencing. Cancer Discov 4:956-971

4. Brat DJ, Aldape K, Colman H et al (2018) cIMPACT-NOW update 3: Recommended diagnostic criteria for "Diffuse astrocytic glioma, IDH-wildtype, with molecular features of glioblastoma, WHO grade IV." Acta Neuropathol 136:805-810

5. Le Rhun E, Preusser M, Roth P et al (2019) Molecular targeted therapy of glioblastoma. Cancer Treat Rev 80:101896

6. Vivanco I, Robins HI, Rohle D et al (2012) Differential sensitivity of glioma- versus lung cancer-specific EGFR mutations to EGFR kinase inhibitors. Cancer Discov 2:458-471

7. Pines G, Huang PH, Zwang Y, White FM, Yarden Y (2010) EGFRvIV: A previously uncharacterized oncogenic mutant reveals a kinase autoinhibitory mechanism. Oncogene 29:5850-5860

8. Huang PH, Xu AM, White FM (2009) Oncogenic EGFR signaling networks in glioma. Science Signal 2:re6-re6

9. van den Bent MJ, Gao Y, Kerkhof M et al (2015) Changes in the EGFR amplification and EGFRvIll expression between paired primary and recurrent glioblastomas. Neuro-Oncol 17:935-941

10. Felsberg J, Hentschel B, Kaulich K et al (2017) Epidermal growth factor receptor variant III (EGFRvIII) positivity in EGFR-amplified glioblastomas: Prognostic role and comparison between primary and recurrent tumors. Clin Cancer Res 23:6846-6855 
11. Eskilsson E, Rosland GV, Talasila KM et al (2016) EGFRvIll mutations can emerge as late and heterogenous events in glioblastoma development and promote angiogenesis through Src activation. Neuro-Oncol 18:1644-1655

12. Abou-Fayçal C, Hatat A-S, Gazzeri S, Eymin B (2017) Splice variants of the RTK family: Their role in tumour progression and response to targeted therapy. IJMS 18:383

13. Reardon DA, Desjardins A, Vredenburgh JJ et al (2020) Rindopepimut with Bevacizumab for patients with relapsed EGFRvIIIexpressing glioblastoma (ReACT): Results of a double-blind randomized Phase II trial. Clin Cancer Res 26:1586-1594

14. Weller M, Butowski N, Tran DD et al (2017) Rindopepimut with temozolomide for patients with newly diagnosed, EGFRvIIIexpressing glioblastoma (ACT IV): A randomised, double-blind, international Phase 3 trial. Lancet Oncol 18:1373-1385

15. Higa N, Akahane T, Yokoyama S et al (2020) A tailored next-generation sequencing panel identified distinct subtypes of wildtype IDH and TERT promoter glioblastomas. Cancer Sci 111:3902-3911

16. Jonsson P, Lin AL, Young RJ et al (2019) Genomic correlates of disease progression and treatment response in prospectively characterized gliomas. Clin Cancer Res 25:5537-5547

17. Cerami E, Gao J, Dogrusoz U et al (2012) The cBio Cancer Genomics Portal: An open platform for exploring multidimensional cancer genomics data: Figure 1. Cancer Discov 2:401-404

18. Gao J, Aksoy BA, Dogrusoz U et al (2013) Integrative analysis of complex cancer genomics and clinical profiles using the cBioPortal. Science Signal 6:pl1-pl1

19. Kikuchi Z, Shibahara I, Yamaki T et al (2020) TERT promoter mutation associated with multifocal phenotype and poor prognosis in patients with IDH wild-type glioblastoma. Neuro-oncol Adv 2:vdaa114

20. Umehara T, Arita H, Yoshioka E et al (2019) Distribution differences in prognostic copy number alteration profiles in IDH-wildtype glioblastoma cause survival discrepancies across cohorts. Acta Neuropathol Commun 7:99

21. Lee JC, Vivanco I, Beroukhim R et al (2006) Epidermal growth factor receptor activation in glioblastoma through novel missense mutations in the extracellular domain. PLoS Med 3:e485

22. Mok TS, Wu Y-L, Ahn M-J et al (2017) Osimertinib or platinum-pemetrexed in EGFR T790M-positive lung cancer. N Engl J Med 376:629-640

23. Fukushima T, Favereaux A, Huang $\mathrm{H}$ et al (2006) Genetic alterations in primary glioblastomas in Japan. J Neuropathol Exp Neurol 65:12-18

24. Santarpia M, Liguori A, Karachaliou N et al (2017) Osimertinib in the treatment of non-small-cell lung cancer: Design, development and place in therapy. Lung Cancer (Auckl) 8:109-125

25. Yver A (2016) Osimertinib (AZD9291) - a science-driven, collaborative approach to rapid drug design and development. Ann Oncol 27:1165-1170

26. Makhlin I, Salinas RD, Zhang D et al (2019) Clinical activity of the EGFR tyrosine kinase inhibitor, Osimertinib, in EGFR-mutant glioblastoma. CNS Oncology 8:CNS43

27. Christians A, Adel-Horowski A, Banan R et al (2019) The prognostic role of IDH mutations in homogeneously treated patients with anaplastic astrocytomas and glioblastomas. Acta Neuropathol Commun 7:156

28. Yoshimoto K, Dang J, Zhu S et al (2008) Development of a real-time RT-PCR assay for detecting EGFRvIll in glioblastoma samples. Clin Cancer Res 14:488-493

29. Zacher A, Kaulich K, Stepanow S et al (2017) Molecular diagnostics of gliomas using next generation sequencing of a gliomatailored gene panel: Next generation molecular diagnostics of gliomas. Brain Pathol 27:146-159

30. Jeuken J, Sijben A, Alenda C et al (2009) Robust detection of EGFR copy number changes and EGFR variant III: Technical aspects and relevance for glioma diagnostics. Brain Pathol 19:661-671

31. Mehta Al, Persson O, Herndon JE et al (2011) Reply to M.S. Lesniak. J Clin Oncol 29:3105-3106

32. Del Vecchio CA, Giacomini CP, Vogel $\mathrm{H}$ et al (2013) EGFRvlll gene rearrangement is an early event in glioblastoma tumorigenesis and expression defines a hierarchy modulated by epigenetic mechanisms. Oncogene 32:2670-2681

\section{Tables}

Table 1. High grade glioma cases with EGFR gene alterations

Page $7 / 11$ 


\begin{tabular}{|c|c|c|c|c|c|c|c|c|c|c|}
\hline $\begin{array}{l}\text { Research } \\
\text { ID }\end{array}$ & Pathology & Sex & Age & SNV & CNV & & $\begin{array}{l}\text { Vaf } \\
(\%)\end{array}$ & impact & $\begin{array}{l}\text { mutation } \\
\text { effect }\end{array}$ & $\begin{array}{l}\text { evidence } \\
\text { type }\end{array}$ \\
\hline 2 & GBM & male & 81 & p.A289D & 19.43 & amplification & 80 & missense & $\begin{array}{l}\text { gain of } \\
\text { function }\end{array}$ & - \\
\hline \multirow[t]{2}{*}{8} & \multirow[t]{2}{*}{ GBM } & \multirow[t]{2}{*}{ female } & \multirow[t]{2}{*}{70} & p.S229C & \multirow[t]{2}{*}{1.76} & \multirow[t]{2}{*}{ intact } & 28.9 & missense & $\begin{array}{l}\text { gain of } \\
\text { function- } \\
\text { predicted }\end{array}$ & - \\
\hline & & & & p.G588D & & & 43.4 & missense & unknown & - \\
\hline \multirow[t]{2}{*}{83} & \multirow[t]{2}{*}{ GBM } & \multirow[t]{2}{*}{ male } & \multirow[t]{2}{*}{63} & p.V774M & \multirow[t]{2}{*}{29.40} & \multirow[t]{2}{*}{ amplification } & 75.4 & missense & unknown & - \\
\hline & & & & p.T790M & & & 80.5 & missense & $\begin{array}{l}\text { gain of } \\
\text { function }\end{array}$ & $\begin{array}{l}\text { druggable } \\
\text { mutation }\end{array}$ \\
\hline 85 & GBM & male & 78 & p.L747A & 2.48 & intact & 19.3 & missense & unknown & - \\
\hline \multirow[t]{3}{*}{93} & \multirow[t]{3}{*}{ GBM } & \multirow[t]{3}{*}{ female } & \multirow[t]{3}{*}{65} & p.R108K & \multirow[t]{3}{*}{20.79} & \multirow[t]{3}{*}{ amplification } & 33.2 & missense & $\begin{array}{l}\text { gain of } \\
\text { function }\end{array}$ & - \\
\hline & & & & p.A289V & & & 24.17 & missense & $\begin{array}{l}\text { gain of } \\
\text { function }\end{array}$ & $\begin{array}{l}\text { druggable } \\
\text { mutation }\end{array}$ \\
\hline & & & & p.D984Y & & & 83.37 & missense & unknown & - \\
\hline 96 & GBM & male & 51 & p.S442I & 2.00 & intact & 19.3 & missense & unknown & - \\
\hline 97 & GBM & male & 73 & p.F254l & 1.98 & intact & 29.39 & missense & unknown & - \\
\hline 19 & $A A$ & male & 49 & p.R108K & 10.49 & amplification & 70 & missense & $\begin{array}{l}\text { gain of } \\
\text { function }\end{array}$ & - \\
\hline 52 & AA & female & 71 & p.A289D & 104.10 & amplification & 12 & missense & $\begin{array}{l}\text { gain of } \\
\text { function }\end{array}$ & - \\
\hline 53 & AA & male & 61 & p.A289T & 35.77 & amplification & 42.5 & missense & $\begin{array}{l}\text { gain of } \\
\text { function }\end{array}$ & - \\
\hline \multirow[t]{2}{*}{59} & \multirow[t]{2}{*}{ AA } & \multirow[t]{2}{*}{ male } & \multirow[t]{2}{*}{67} & p.G598V & \multirow[t]{2}{*}{2.57} & \multirow[t]{2}{*}{ intact } & 23.6 & missense & $\begin{array}{l}\text { gain of } \\
\text { function }\end{array}$ & - \\
\hline & & & & p.S768I & & & 19.2 & missense & $\begin{array}{l}\text { gain of } \\
\text { function }\end{array}$ & $\begin{array}{l}\text { druggable } \\
\text { mutation }\end{array}$ \\
\hline 90 & $A A$ & male & 39 & p.Т790M & 5.52 & amplification & 28.1 & missense & $\begin{array}{l}\text { gain of } \\
\text { function }\end{array}$ & $\begin{array}{l}\text { druggable } \\
\text { mutation }\end{array}$ \\
\hline
\end{tabular}

Table 2. Repertoire of EGFR variant detecting in high grade glioma cases

\begin{tabular}{|c|c|c|c|c|c|c|}
\hline Research ID & Pathology & Sex & Age & Type of variants & CNV & \\
\hline 15 & AA & female & 57 & EGFRv $\rrbracket$ & 42.1 & amplification \\
\hline 16 & GBM & female & 60 & EGFR exon 2-14 del & 25.5 & amplification \\
\hline 21 & GBM & female & 82 & EGFR exon 2-14 del & 58.9 & amplification \\
\hline 28 & GBM & male & 62 & EGFRv $\rrbracket$ & 52.8 & amplification \\
\hline 49 & GBM & male & 64 & EGFRv $\rrbracket$ & 169.4 & amplification \\
\hline 112 & GBM & male & 77 & EGFR exon 6-7 del* & 165.1 & amplification \\
\hline 113 & GBM & male & 72 & EGFRv》 & 82.2 & amplification \\
\hline
\end{tabular}

*:novel EGFR variant 


\section{Figures}

a
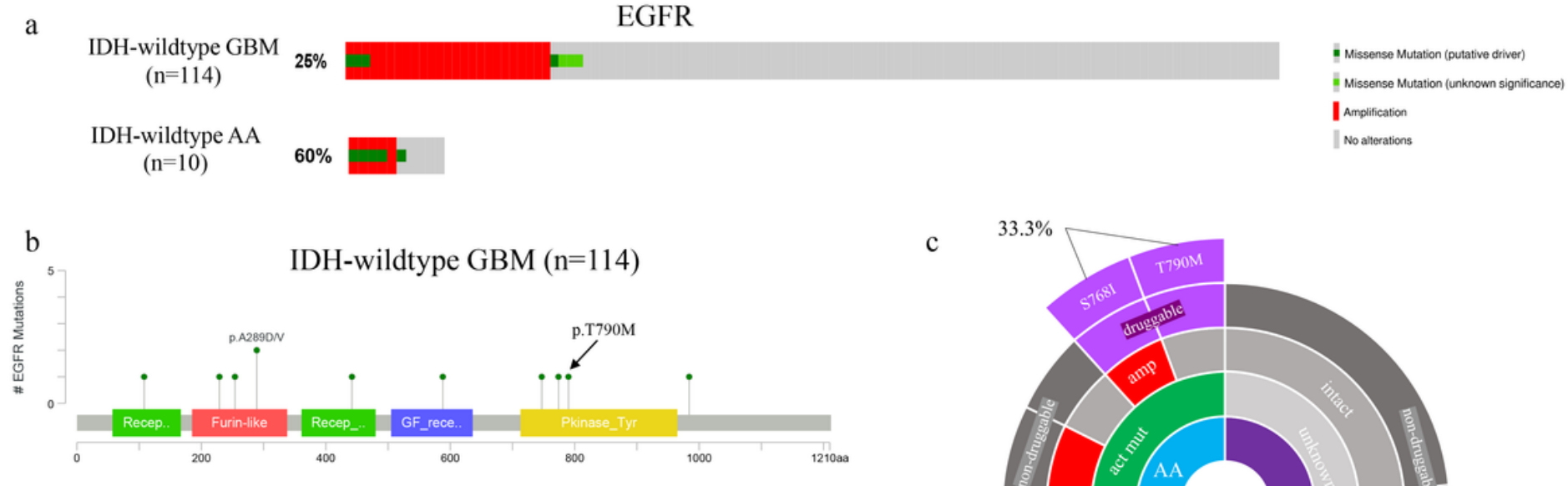

$\mathrm{c}$
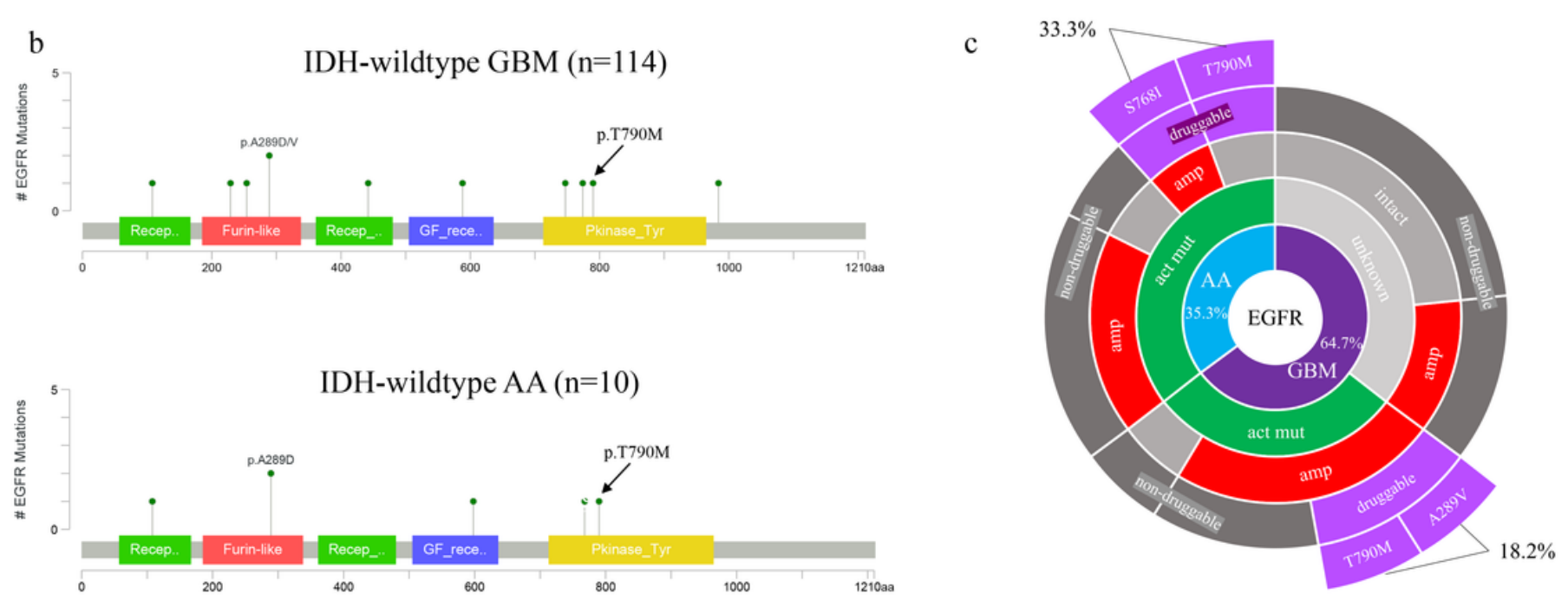

Figure 1

Somatic alterations of EGFR. (a) EGFR mutations and copy number alterations were generated and visualized using OncoPrinter included in the cBioPortal for Cancer Genomics software suite. (b) EGFR protein domain structure with somatic mutations summarized from 114 IDH-wildtype GBMs and 10 IDH-wildtype AAs. (c) Sunburst chart showing the EGFR mutation spectrum in IDHwildtype GBMs and IDH-wildtype AAs. Percentages were calculated from the total EGFR mutation sites 

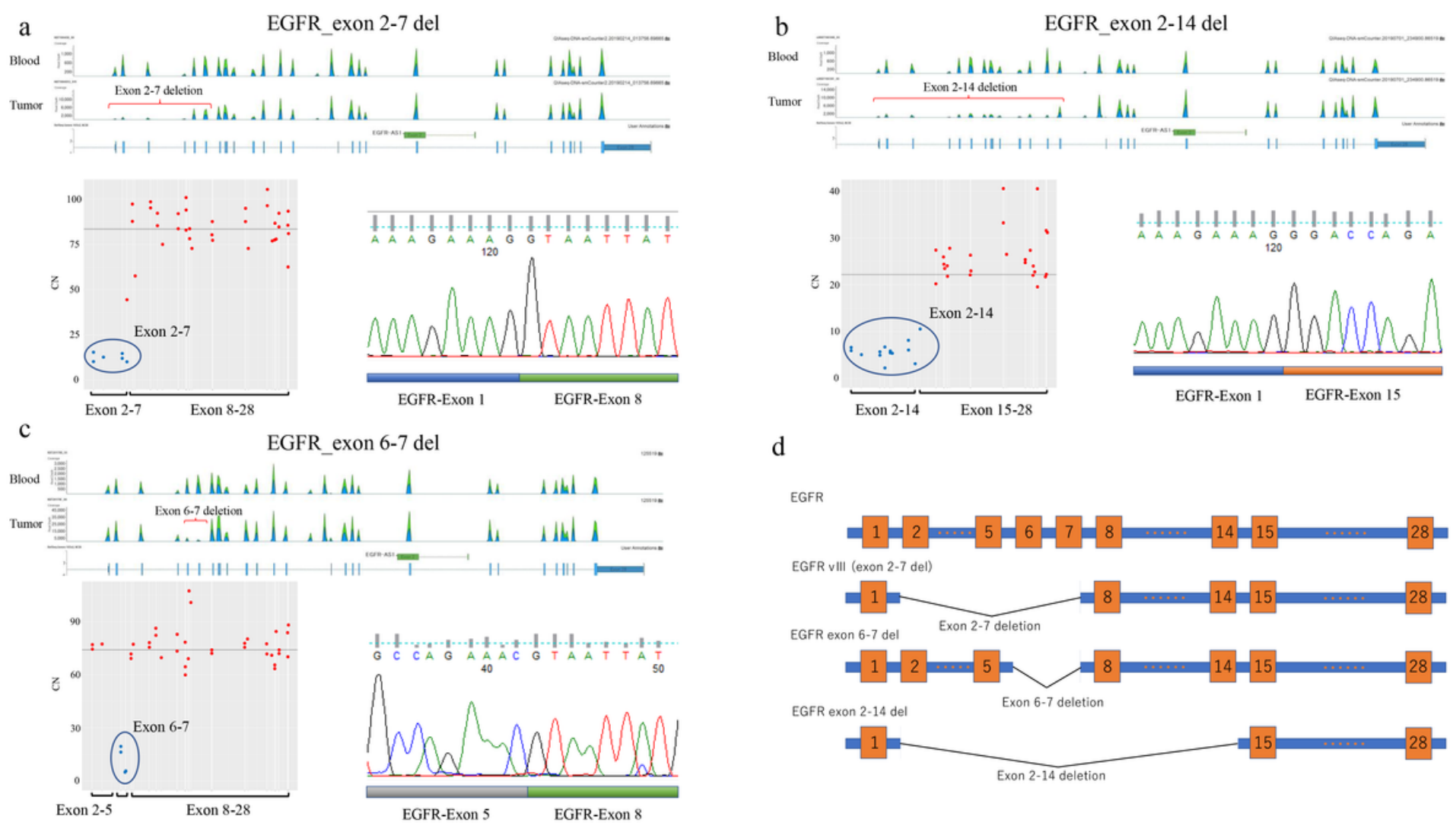

d

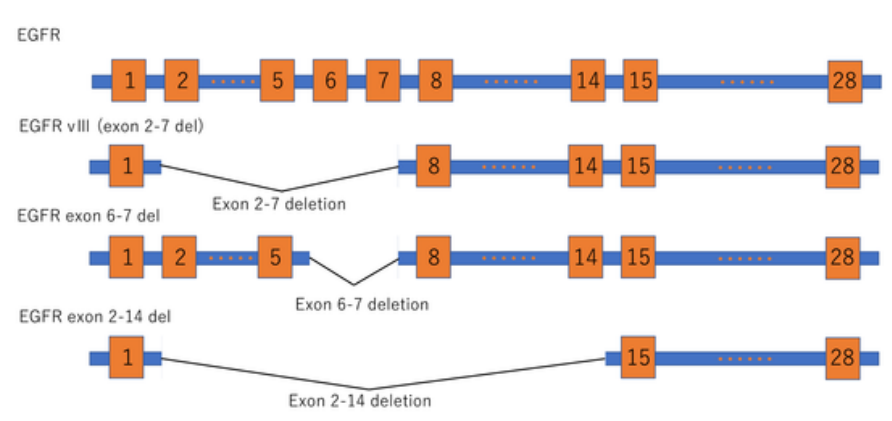

Figure 2

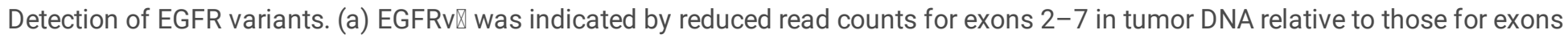
2-7 in matched leukocyte DNA (upper panel) and was detected based on reduced CN of exons 2-7 compared with the average CN

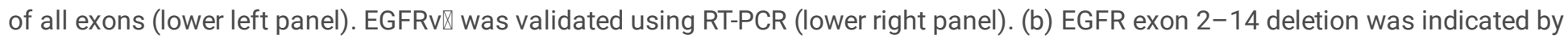
reduced read counts for exons 2-14 in tumor DNA relative to those for exons 2-14 in matched leukocyte DNA (upper panel). It was detected based on reduced CN of exons 2-14 compared with the average CN of all exons (lower left panel). EGFR exon 2-14 deletion was validated using RT-PCR (lower right panel). (c) EGFR exon 6-7 deletion was indicated by reduced read counts for exons 6-7 in tumor DNA relative to those for exons 6-7 in matched leukocyte DNA (upper panel). It was detected based on reduced CN of exons 6-7 compared with the average $\mathrm{CN}$ of all exons (lower left panel). EGFR exon 6-7 deletion was validated using RT-PCR (lower right panel). (d) Structures of EGFR and its splicing variants. EGFRv (exon 2-7 deletion), exon 6-7 deletion, and exon 2-14 deletion are shown 
a
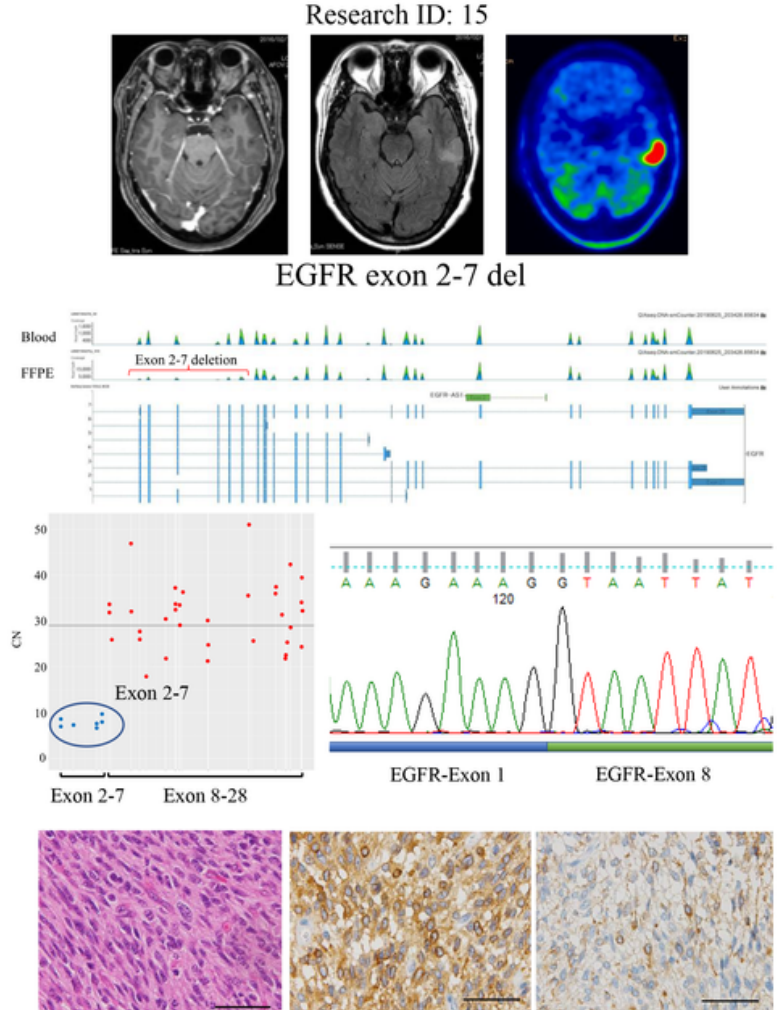

HE staining

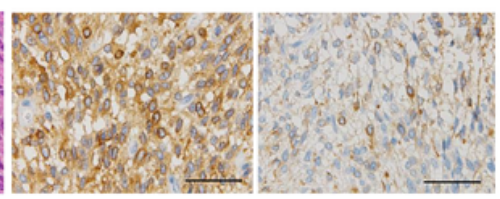

EGFR wild

EGFR vIII b
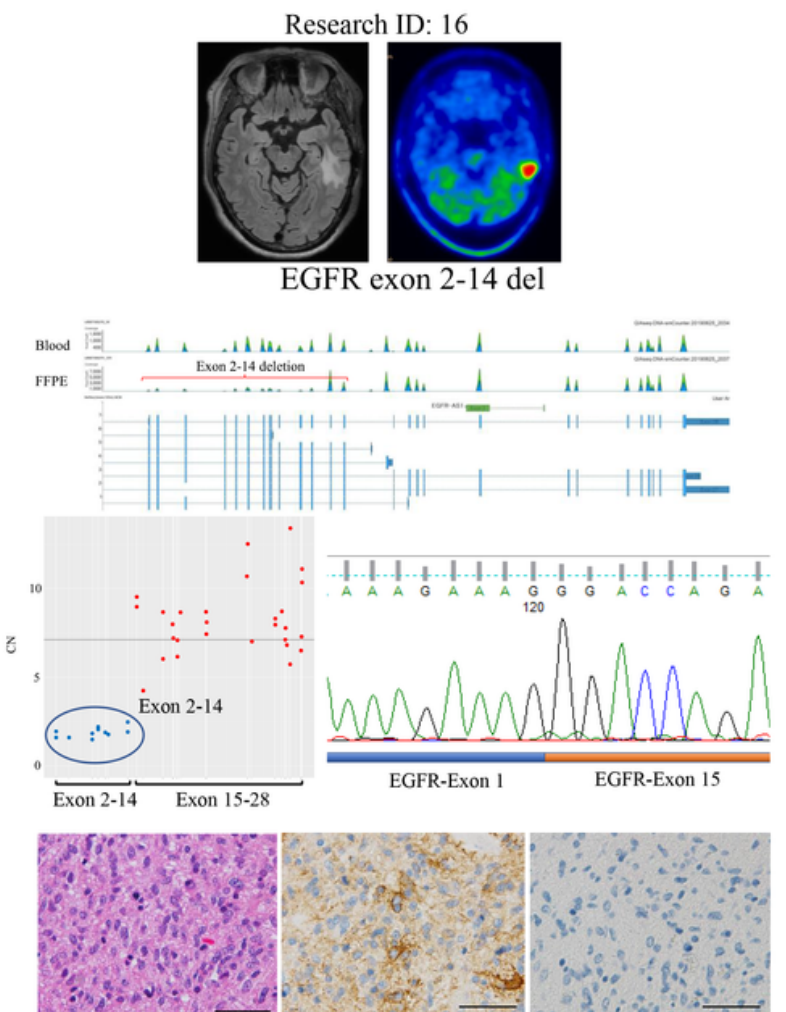

HE staining

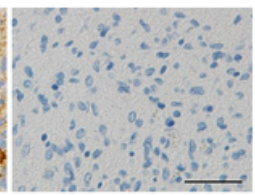

EGFR vIII

Figure 3

Longitudinal transformation of EGFRv . (a) Upper: Enhanced T1WI, FLAIR, and methionine PET in an initial GBM case with IDHwildtype AA. Middle: EGFRv $\$ detected by NGS and RT-PCR. Lower: Hematoxylin, eosin, and immunostaining of EGFR wildtype and EGFRv囚. (b) Upper: FLAIR and methionine PET in a recurrent GBM case with IDH-wildtype GBM. Middle: EGFRv detected by NGS and RT-PCR. Lower: Hematoxylin, eosin, and immunostaining for EGFR wildtype and EGFRv

\section{Supplementary Files}

This is a list of supplementary files associated with this preprint. Click to download.

- Supplementarytable.S1.xIsx

- supplementaryfig.1.pdf 